\title{
Adaption Of Construction Management Modelling System Through The Use Of Internet Of Things (IoT)
}

\author{
Dr. Ali Bakhit Jaafreh (Associate Professor) \\ $\mathrm{PhD}$ Management Information Systems \\ Shaqra University, Business Department, KSA \\ Eng. Mohammad B. Al Jaafreh \\ Mechanical Engineer, Dar AL Handasah Co., Makkah, SA
}

Doi:10.19044/esj.2021.v17n29p263

Submitted: 07 June 2021

Accepted: 01 July 2021

Published: 31 August 2021
Copyright 2021 Author(s)

Under Creative Commons BY-NC-ND

4.0 OPEN ACCESS

Cite As:

Jaafreh A.B. \& Al Jaafreh M.B. (2021). Adaption Of Construction Management Modelling System Through The Use Of Internet Of Things (IoT). European Scientific Journal, ESJ, 17(29), 263.

https://doi.org/10.19044/esj.2021.v17n29p263

\section{Abstract}

This paper aims to adapt, implement, and practice the physical use of IoT in the construction management industry as a configuration protocol. The authors describe the general outlines of this field capability as a framework, and they foresaw the opportunities of developing a dominant steam line approach to be incorporated in the construction management side. The challenge is to bond the BMS, SCADA, IoT, smart data "Tags", green building concepts, smart surveillance, self-moving transportation, and mobile technology in one practical, innovative configuration model. The authors, however, called it "Construction management modelling system" (CMMS). The gap point in this paper is the harnessing of Data Tagging systems along with a smart mobile system via radio frequency and Wi-Fi communication system. The significant effort here is to establish optimum modelling of the IoT system to roll out a product containing all the solutions for the existing and expected obstacles in the construction sites for the different types of projects. The literature review, proposed configuration system, affiliate works, benefits, conclusions, and recommendations have been furnished.

Keywords: Internet Of Things, Smart Data, Construction Management; Tags, RFID, Sensors 


\section{Introduction}

The expression "Internet of Things” (IoT) was coined by Kevin Ashton as a description of a series of integrated communications of the physical objects of the real world through the Internet (Ashton, 2009). By definition, the interconnection embedded in everyday objects via the internet of computing devices enables them to send and receive data. The Internet of Things (IoT) is the case where things interacted with each other with a significant common nature identified, sensed, data given and communicated. In the construction world, megaprojects are incredibly full of trades, equipment, building materials, supplies, moving items, sensing items, peoples, types of machinery, and vehicles. Therefore, it is the best environment for the demonstration of a new proposed system (Construction Management Modeling System (CMMS)). Based on a study carried out by Turner and Townsend for the International construction market survey 2018 (McGuckin, 2018), there is a $4 \%$ rise in construction cost and $60 \%$ skill shortages in global markets. Therefore, the IoT offers to minimize the increase in construction cost and replace/compensate for the skill shortages. To understand the importance and effect of the IoT weight in the market, Gartner's forecasted that "about $20 \%$ or 1.37 Billions of IoT units shipped into smart city applications by 2020 will be utilized for sustainability and climate change use cases, especially in the transportation and smart buildings, as well as homes." The number of devices connected to the internet is expected to be 50 billion by 2020 (Gartner, 2019). McKinsey research in 2018 predicted that, "By 2020, the smart city industry is estimated to reach a $\$ 400$ billion market, with 600 cities worldwide. These cities are expected to generate $60 \%$ of the world's GDP by 2025” (McKinsey, 2018). Based on the above, the combination of construction management and IoT in one binding transformation will be a promising market with vast prospects. It is defined as a "smart city" where all the data were collected and utilized purposely to fulfil the activities' functions during the periods of building and demolishing. The area of the "smart city" concept is interconnected with the prediction of weather, traffic, crime, and temperature parameters (Kyriazis et al., 2013). Consequently, IoT can help reduce the cost of energy, spatial management, and building maintenance by up to $30 \%$ especially in large sites, such as: industrial zones, office parks, shopping malls, airports or seaports (Gartner, 2016).

Today, there are many examples of applications for this invention (IoT). Thus, it is used in Manhattan Bridge in New York, where they measure the temperature, humidity, strains, and corrosion through the Web portal. The United States started issuing the new e-passport in 2007, which has a chip embedded in the last page of the passport holding digitally all information needed about the holder. The traveler's suitcase can be checked remotely by scanning and reading the "Tags" attached to its item. The new technology the 
apple company has started now is the "Apple Tag", which is related to the search for lost things having a "Tag" embedded inside. Also, it strengthens the airdrop quality. Google Glass is another example. It is to be used by workers in dangerous locations and needs a free hands mode. Also, it is to be used in equipment visors and vehicle windshields to work remotely, and send directions and information by augmented reality. It can also equip sensors in the constructed building connecting them to the "Building Information Modeling (BIM)" to give direct real life of construction. These sensors can send back information on how the materials are affected by age and climate changes. Changes in energy efficiencies in roofing, walls, doors and windows can be sent back to the BIM and analyzed. Besides, how structures behave in case of earthquakes or how bridges bend under the weight of massive traffic (Kensek, 2014) has shown that the best way of utilizing the optimum helpful result in the construction industry is by using the Radio Frequency Identification signals (RFID). The RFID can be achieved through a vast number of methods and technologies, including Near Field Communication (NFC), Infrared (IR) sensors, and many more (Chan, 2015).

The big picture here is looking to the future and viewing the light of decades from now. "Things" are objects which are measured, sensed, monitored, and controlled by a software system with feedback controlling units. The construction side "Things" is counted the same as household "Things" because they are somehow connected in the building world. There is an understanding of the reluctance of the society and stakeholder's bureau to accept the innovations and new technology for the first time. However, the paper here encourages them to understand and minimize the rejection of the latest technology to avoid waste of time as it is dominating the construction yard and recent era (Jaafreh, 2018). Since construction management is not utilizing the full IoT features in the construction sector because of the lack of configuration protocols, the authors suggest using the "Construction Management Modeling System" (CMMS). There is a need to step forward and enhance efforts to prepare the environment where IoT can be used in construction industry management without any obstacles with the relevant regulation and legislations.

The authors tried in this paper to focus on a specific process, i.e., Construction Management System (CMS), by using the IoT and neglecting the unwanted surrounding parameters that are varying with time and changing alternatively. This is because it is a large-scale field and it needs extra efforts to conclude on the whole aspects of the advantages it possesses. Another helpful motivation nowadays is the "Green Building Concept", which can be a supportive arm to the new proposed CMMS as the construction trend is now ongoing with the LEED (Green Building). Hence, this gives environmental benefits and high sales capability. Green building contains energy efficiency 
strategies, renewables, site selection, water conservation, indoor resource conservation and environmental quality. Green building's vital support is gathering and recording data from the starting of the design phase to the demolition phase. Also, it is creating the best present community and keeping the future generations' rights of natural resources (Thebig5, 2019). All of these are enhancing sustainability and performance. The "LEED" buildings are more likely less harmful to the environment, ecosystems, and biodiversity because they are initiated from the scratch on that basis. The first item in the green building's concept is the sustainability of the system and the synergy between the quality of life, environmental surrounding, and continuous development.

\section{Theoretical Background}

The authors developed the proposed conceptual framework of the "Construction Management Modeling System" (CMMS) based on Information Modeling (IM) and complemented it by a critical review of numerous related articles. An information model is a representation of concepts, relationships, constraints, rules, and operations to specify data semantics for a chosen domain of discourse. The advantage of using an information model is that it can provide a shareable, stable, and organized structure of information requirements for the domain context (Lee, 1999). One of the most crucial things nowadays is the conservation of resources as its abundance is becoming rare. The cost, quality, efficiency for workers, tools, and productivity will not be more achievable, reliable, and easily handled. As long as the range of IoT application is widely vast, construction management will enter the new revolutionary era by using these "Things" and connecting them. Connectivity can be done through the web portal for the fixed items and wireless items through RF for moving items. Most equipment, appliances, objects, and other components used in the construction industry can have sensors to monitor and control the operating conditions. Therefore, collected data should be recorded, processed, and tracked remotely. In addition, feedback should be sent through the same channel (Mohammed \& Elmustafa, 2017).

The project or building is going to be a data-driven process identity. The data pulled out from sensors will help project facility managers to form a sound operational background and give them the ability to make the adjustment and repair capability (Becerik et al., 2012). Lighting powers, fuel consumed, drilling time, and machines running hours are good examples of data collected through the IoT. There will be a swift record of materials used and installation guidance to enhance and shorten the maintenance and repair time. Built-in analytics can investigate the uneven patterns or unusual behavior in the collected data and make the healing response and adjustment 
in real-time. The necessary conditions for this technology to follow up in future is that the components should be an open-source protocol, should be able to communicate with each other, and should be linked to the central control system. It is known that most of the construction sites are sophisticated to customize the standardization of IoT applications because of the variety of variables of related work (Vermesan \& Friess, 2014). The IoT gives the stakeholders, owners, and facilities managers the option to get the whole data needed and the analytics to help achieve better management decision making (Brous et al., 2018). A Building Management System (BMS) is a computerbased system and is known as a control system that is used to monitor and manage mechanical, electrical, and electro-mechanical services. Numerous services included are: air-conditioning power, heating, ventilation, elevators and lights, physical access control, and pumping stations (Reddy, 2011). Building Management Systems (BMS) are also known as Building Management and Control System (BMCS), Building Automation Systems (BAS), and Direct Digital Controls (DDC). The purpose of BMS is to deliver a complete solution for the life cycle of the building environment from the design stage to construction and operation.

SCADA systems control distributed assets where centralized data acquisition is as important as control (Rao et al., 2017). SCADA systems include waste water collection systems, water distribution, electrical utility transmission and distribution systems, oil and gas pipelines, and public transportation systems. SCADA systems incorporate data acquisition systems with data transmission systems and HMI software to deliver a centralized monitoring and control system for several process inputs and outputs. SCADA systems are designed to collect field information, transfer it to a central computer facility, and display the information to the operator graphically or textually. This therefore allows the operator to monitor or control an entire system from a central location in real time (Rao et al., 2017). This study extensively contributes to existing knowledge on using digital technologies to synthesize the existing IoT literature and to understand the IoT. Building on this knowledge, the authors will provide a theoretical framework and conceptual model, including the IoT adoption and implementation guide for stakeholders and directions for future research.

\section{Conceptual Framework}

The Building Management System (BMS) and the Supervisory Control and Data Acquisition (SCADA) System are two systems primarily used in electro-mechanical works, functional if integrated with the IoT. The authors suggest a module from these three systems to apply in the Construction Management System (CMS). The combination of IoT, BMS, SCADA, and smart data will produce the "continuous feedback" system for the proposed 
construction management system (Construction Management Modeling System (CMMS)). The primary goal is to make the construction management industry self-configured, self-optimized, self-diagnosed, and fully independent with its configuration protocol. The CMMS is a continuous learning revolutionary process aiming to unify, monitor, respond, control, and manage the data taken from the site or people and machines to prototype a successful smart building. Most of the tools used here are transmitters, sensors, meters, appliances and devices, which can receive and send data about their current physical state and the surrounding environment through the internet. Most of the features used here are the RFID and Mobile Wi-Fi signals.

The authors did not discuss the BMS and SCADA inputs and consider them as black box provider to complete the integration of the construction management module. Therefore, the base is ready, but the exhaustive combination of all-in-one basket is the crucial task to carry out. The authors will suggest a starting module as a trial since the task needs more effort and more mutual work to be initiated and established as a complete detailed package. This paper suggests opening the window of producing a "united module" of IoT, BMS, and SCADA systems to apply in the construction management side.

- Proposed model: CMMS (Construction Management Modeling System)

- Application: High-rise towers, megaprojects, and LEED/iconic projects/buildings

- Area of Interest: Stakeholders, ownership, management, design environment, manufacturing processes, procurement and supply chain, installations, operations, jurisdictions, and disposal.

\section{The Construction Sector in KSA}

Taking a deep look at the construction sector side in KSA, it will be astonishing to find a significant number of billions of dollars covering an entire umbrella of diversity of projects leading the development process among all of Saudi Arabia's entire land. The construction sector is one of the largest sectors that contribute to the national economy. It is overgrowing in Saudi Arabia, where the total value of projects in the construction sector during 2017 was about 162 billion dollars. 103 billion dollars are governmental projects. The sector's operating income was 57 billion dollars in 2017 (General Authority for Statistics, 2017). Large-scale projects such as NEOM with the projected value of more than $\$ 500$ billion, which covers 26,500 square kilometers in Tabouk along with thousands of other megaprojects under construction across the country like Haramin projects in Makkah, Madinah, and Aramco company projects, will increase demand for innovative construction solutions in Saudi Arabia (https://www.neom.com/). 
Nobody can imagine the ultimate returns/benefits of using such a proposed system and the easiness of providing the total exemptions in labor, deliveries, and time. Based on the above, it is worthy to develop such a protocol that will bring about cost savings in energy, labours, materials, and in maintaining quality and time.

\section{Construction Management Phases}

The IoT helps in the construction phase as well as the completed building phase.

\section{Construction Phase}

Smart building (IoT \& Construction Management) can shorten the construction period from the planning to the completion of the construction by a significant figure, likely from 3-5 years to half of that (KPMG, 2016). The authors are aware that only large companies or mega projects can apply the IoT during the design, construction, and operational phase. It can also work with iconic projects. However, as time passes, the issues will be more familiar and more comfortable, where this situation will expand dramatically and internationally. Most expertise anticipates that the cost will increase by applying the intelligent building concept (IoT) and are going to be 15\%-30\% based on the level of "smartness" of the constructed building (Urie, 2019; Bashir \& Gill, 2016). Something to remember here is that energy savings are around 30\% continuously (Urie, 2019). The smartness of the constructed building will vary from "basic" innovative hardware, "selective" smart hardware to "fully" smart hardware based on the awareness of the stakeholders, and environmental sustainability understanding. From the current development continuation process, it will reach the "basic" category very soon because it is solving many problems and easing the communication process between the governmental and municipal sectors and the people.

An excellent example of that is the Google London headquarters at King's Cross, which scratched from the planning phase to be the most innovative building on earth. This method or procedure reduces the activity execution time, cost of extra quantities to cover the delay and unorganized procurement used in the old traditional way, and this would result to the finishing of project on time (Jia et al., 2017). Here are some examples of the applications, which show the evolutionary change of doing work: By many ways as labelling, the significant units of supply on-site are being "tagged". As a result, the system can follow and count them, and when the storage comes down to the prefixed level, the system orders more from the central storage warehouse. Tracking the equipment and tools helps to minimize the time wasted in finding the equipment. It also reduce the project's cost by reducing the replacement of the devices (Wang et al., 2016; Oriwoh et al., 2013). Other 
examples include the monitoring of the construction equipment and vehicle fleet locations through the GPS, and the tracking of construction cranes and heavy landscaping machinery (Urie, 2019; Hamilton et al., 2017).

Linking the preventive maintenance program with the vehicles and machines used in the project will save time, cost, and enhance the reliability of work to be finished on time. IoT can help construction companies in tracking their labours on site and ensuring labours' safety by using wearable technology (Woodhead et al., 2018; Wan et al., 2016). Wearable technology can give a headcount of the site workers and their current distribution at the site and provide emergency alerts for all the worksite workers (Khakurel et al., 2018). They are minimizing the risk management to the lowest value due to transparency, pre-detective procedures of problem-solving, and availability of data (NIST, 2019). Project planning and project schedules are going to be more realistic and reliable since these planning and schedules are produced directly from a real-time deliverable. Collecting the data from various aspects gives the management outstanding potentials and options to make the right decisions.

\section{Operational Phase}

Smart Building Management Systems have the ability to interact in real time through learned action and by using the recorded information collected by the sensors to adjust any deviated data or preset value regulations (Mataloto et al., 2019). Thus, this includes shutting down the A/C in non-used rooms/areas or adjusting the flow of air based on the occupancy level. Another example is the weather forecast data, which have to be addressed to provide automated directions to the running equipment to act responsively to adjust their performance accordingly, like the quantity of external air to enter the building and the infiltration rate. The collected/recorded data of the building is a valuable store material and might be used statistically to make modelling for the building performance, occupancy behaviors, and user experiences. The collected/recorded data can be used in the design stage for future projects and develop reliable construction and usage patterns (Oduyemi \& Okoroh, 2016). Building owners can choose to make the intelligent system with open-source protocol such that they can provide the "basic" and keep the choice for the tenants to add up "Things" as per their requirement. Nonetheless, the main parameter here is the capacity of the software to contain, digest, and cope with future data.

An Increase in "Things” connectivity leads to complexity in real life, which needs new approaches to mitigate the affluence of such smart products. Things value can be compared based on their intelligence and advanced analytics capabilities. Due to the usage of wireless RF signals, the savings in structured cabling and their accessories are highly considerable. The rent value 
of the smart building would be rise around 3\%, and the equity value will be around $10 \%$ (OSRAM, 2017; Eggleton, 2020). Another subsidiary benefit can come from selling the data of people during the construction phase or the completed building phase as "analytics service" to the advertisers and developers for their future projects. All the electromechanical works will assemble through BMS and SCADA systems and enter directly to the proposed primary central protocol, "Construction Management Modeling System” (CMMS), through configuration matches. No need to go into details any further in electromechanical systems because they have their data systems and need just protocols configuration to link to the proposed method (CMMS).

The benefits of IoT to the completed project/building are summarized as follows:

- Preventive maintenance, which saves a lot of money, time and quality.

- The ability of all stakeholders/facilities managers to control the building remotely, the easiness of extracting the data, specifications and brands models of all materials used in the building through the construction time and usage time, and the high resale value of the building because it is well-known with a open component trusty.

\section{The Collected Data Mining}

- The stress, the strain, and the constructed post-tension data can monitor, control, and justify the building's lifetime.

- The building performance, pointing inefficiencies, and automatic adjustment can provide the corrective actions.

- The temperature, humidity, wind, weather conditions, noise levels, vibrations, Co2, acoustics, water flow, and corrosion rate.

- The project security cameras data (monitoring remotely).

- The project safety cameras data (monitoring remotely).

- Water leakage in hidden zones by piezometers.

- The equipment and machinery data (working hours, fuel consumption and maintenance dates).

- The workers' health, alertness, and working conditions to avoid heat stress, fatigues and reduce site accidents.

- Monitor and control after work lighting levels to save energy of cameras and sensors.

- The supply units tagging data.

- The central storage quantity data and comparable site quantity data.

- The construction equipment and vehicle fleet locations through GPS.

- The replacement time for particular equipment parts or components.

- The unjustified cost of operating equipment out of normal conditions. 
- Pictures and views of the project through fixed cameras (during the construction period) to measure progress and evaluate the construction completion percentages.

- The cost of unscheduled downtime when a machine breaks recording.

- Materials, delivery, specification, and brands names records used in construction phase record as references.

- Operational maintenance, preventive maintenance and repair work programs.

- BMS and SCADA data in conjunction with BMS/SCADA systems through a specific protocol configuration.

Therefore, the authors provide a diagram as the preliminary skeleton of the proposed "CMMS" to start the potential protocol.

Figure 1. Construction Management Modeling System (CMMS) Algorithm (by Authors)

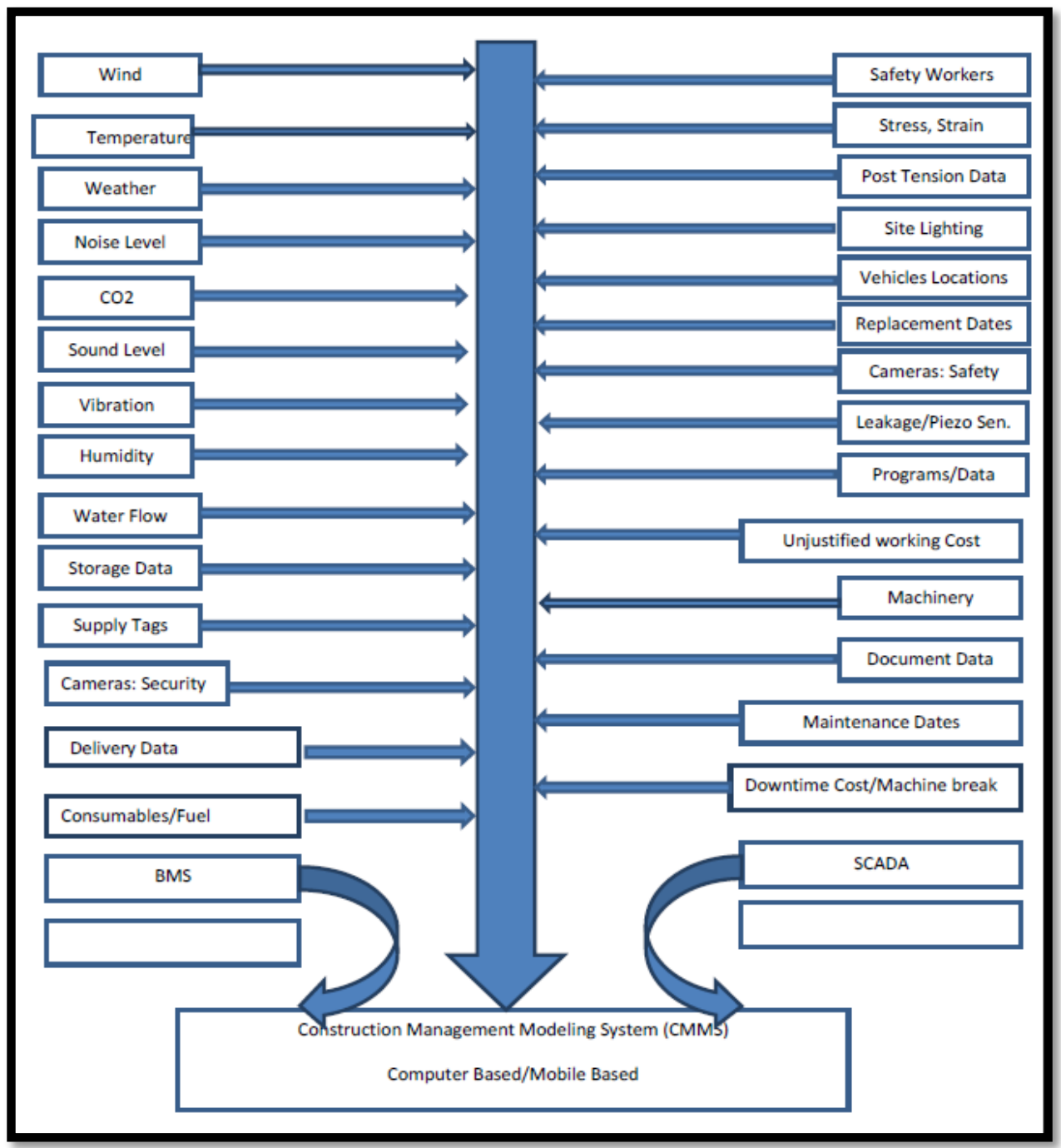




\section{Affiliate Work \\ Methodology and Platform}

This study presents a mixed-methods systematic review of the various areas of application of IoT in modern construction methods. The Modern Method of Construction (MMC) is sometimes referred to as smart construction because it involves streamlining construction processes through increased use of big data, adopting lean principles, digital collaboration, and remote monitoring (Woodhead et al., 2018). The mixed-methods systematic review approach was adopted to enable the investigation of the significant findings from existing literature. Construction management is a massive and multitasking process. It is the base for any building project and is on its way to becoming a reality. The main construction management items to address are monitoring and controlling the project's progress in terms of cost, time, and quality (Zhou et al., 2018). Many responsibilities and many different fields (construction, engineering, software, logistic, and legal issues) are included in the area of work. The new technologies will change current methodologies and concepts of the construction world. In a way, there is no awareness of these changes because people are still hanging with the customized ones and not looking seriously at the massive changes in the coming future. It has been easy to admit that the small and medium projects are out of scope because of the extra cost and the additional need for such systems (Cadle \& Yeates, 2008).

The proposed platform should be the same mobile wireless network without a "transmission booster tower" since the project area is limited. The methodology will serve as a continuous feedback and a learning system (Smart Learning). Authors are talking about a vast technological transformation worldwide, starting from "smart cities" and going beyond the "smart countries". They enable vendor's Things or devices to configure on a specific standard level for all protocols used in the field of industry. All "Things" must be controllable, accessible and disposable by their owners. All "Things" with the same owner/operator must have the ability to identify each other spontaneously to avoid rogues or intruders. The old saying, plug and play, should be the key for installing and removing "Things" to/from the "Things" network. All "Things" must have property/owner identification code and should be scanned and or read automatically or by barcode.

\section{Functionality, Reliability and Responsibility}

The mainstream and realistic direction is going with wireless systems as all technologies are going there. Facilitating the opportunity of getting the "LEED" certification (Green Building) quickly, the international trend for LEED projects gives more attractiveness to the IoT concept (Dandekar et al., 2017). Sometimes, the trend goes with the wired connection for stationary and fixed items, and wireless for others. Anyhow, it is a design decision based on 
the local environment of the area of interest. The means to collect data include: sensors (analog, digital, field, data), RF tags (passive, semi-active and active), central data platform, mobile tracking tools, transmitters, equipment/machinery software, GPS, BMS, SCADA, and smart tools, Web portals.

\section{Types of Controls}

The authors wrote on three types of controls to utilize in this field since they had an adhere effect on the proposed system (CMMS) (NIST, 2019). They are:

- Remote Control: This is well known as the order to change the state of action coming through the central platform or the web portal, like the room temperature changing through the web portal or BMS.

- Learned Action: This is where the smart equipment/device takes action by itself. In other words, machine thinking like equipment/machines ordering themselves to do something without the intervention of any external control.

- Interaction: The rapid action needed or alert action prevailed, generally, for the on-time activity like hazards at site and safety issues.

\section{Regulations}

The stakeholders should recognize and understand their rights and responsibilities for ownership, processing, and managing the "Things" (Oriwoh et al., 2013). New regulations or legislation must be issued based on the specified region to accommodate the traditions and customs used in the dominant cultural background.

\section{Matrix of Rights}

There should be a matrix of rights to be enforced to legalize individuals, companies, and government. It points out the right to connect to the system, the right to charge for the provided service, and the right to control and monitor the illegal overturns which might have happened. The governmental side (law enforcement and authorized jurisdictions) and stakeholder side (owners and manufacturers) should be responsible for the control of IoT devices spread out (Tzafestas, 2018).

\section{Security, Privacy and Data Protection}

It was noticed that the new IoT field forced the literature to identify/specify the owner of the "Things" and the responsibility of errors if it occurred by these "Things". What are the limits or constraints for the data/sensors in front of society? The main thing here is the "control" of "Things" ownership and the extent of that (Oriwoh et al., 2013; Bertino, 2016). 
The European Commission set up a "Privacy Impact Assessment" (PIA) as a legal tool to enhance the awareness of the security and privacy of the IoT (Diaz, 2016). Most people from the privacy perspective are anxious about being tracked in the smart building because of the monitoring systems used to collect the mobile signals and tracking accordingly. So, there is a need to solve this by space monitoring, not mobile tracking. It may be useful as a base for all regulations to be issued to govern the whole sector environment. Rolf Weber suggested using "Privacy Enhancing Technologies" (PET) to increase the privacy and security of radio frequency-based systems like IoT (Weber, 2011). Besides, Rolf sets some requirements: resilience to attacks, data authentication, access control, and client privacy to be included as part of the security and privacy in the risk management to use the IoT (Weber, 2011). The primary responsibility from the authors' point of view is the owner, operator, and organization that is running the system using the IoT. The authors recommend setting other rules and legislations that govern the use of IoT, matching with country or region traditions and habits. Public members and public data collected by the IoT system must be protected from misuse, surveillance, promotion shows use, and media/shopping track. There is a necessity to adopt a security extended umbrella to avoid hacking raids and intruder's interruption.

\section{Legal Issues}

The use and spread out of "Things" bounded by legal authorization and identification tags is to formalize the state of abundance. Legislations must be administered and fixed early before discovering that wasting time on late approvals are causing lost benefits and value (Singh et al., 2018). Knowledge of the rights and responsibilities of the stakeholders to their managing, owning, deploying, and processing of "Things" is the primary tool of the wide spreading of this industry (Singh et al., 2018). In most of cybercrime cases, the law is behind and not adapted swiftly with developments going on in the field of the internet. The Computer Misuse Act in 1990 and the Theft Act of 1968 in the UK can serve as a formative tool for the proposed law.

\section{Future Streaming}

In simple words, no one can stop the technological progress, and the construction industry has to stop the traditional "denial case" of all new things and proceed with the life flow direction. Any mental or cultural reluctance to the future streaming of this aspect means that the construction fields are running out of time and not grabbing any tools to survive. It might be used to monitor the seismic action through the nodes measuring the doors/walls of the building to anticipate any alarming signals. Two aspects of construction management consider it significant and hard to control, which is the materials 
and the workers. IoT through CMMS can solve it by using the workers' wearable and materials tagging alongside tracking systems. By collecting all the physical conditions for the desired building or environment, the occupants can assess and decide the wellness performance. The occupant's choice is from a large-scale perspective, which means people-driven patterns that comes up with a profit. Predictive analytics is popping up in the building management system as an intelligent tool in predicting failures, problems, excessive use, and uneven action. It also helps to analyze it and take corrective actions at the end. The limit of human's creativity is the basic understanding of IoT in the construction management system.

\section{Conclusions}

The authors emphasized that the IoT technology leap is the mandatory scope of the future connectivity of "Things", and it provides a cutting edge of work and allocating a configuration framework to adapt the application in the construction industry. When gathering the IoT, construction management, BMS and SCADA systems, the authors believed that it would create a new concept protocol, which governs the whole building industry and contains both the construction phase and the operational phase. IoT usage reduces energy consumptions through the temporary building process and permanent usage stage. The new system makes restoration and renovation easier in the future due to the availability of all data beyond and post-construction. The proposed system by the authors "CMMS" is the same as BMS/SCADA but exhaustive with both construction period and usage period. Also, it includes the civil, infrastructure, architectural, Building Management System (BMS), SCADA system and related works. This proposal is called the "Construction Management Modeling System” (CMMS). Application sectors include: highrise towers, megaprojects, and LEED/iconic projects/buildings. Area of Interest include: stakeholders, ownership, management, design, manufacturing, procurement, installations, operational jurisdictions, and disposal. The algorithm and software prototype will be carried out using Digital Twin Technology (DTT) and will be the successive step in applying/finalizing the proposed "Construction Management Modeling System" (CMMS).

Smart building (IoT \& Construction Management) can shorten the construction period from the planning to the completion of the construction by a significant figure and record/store the project construction period and operational period data historically. This is so essential for the ongoing operation of the building and the maintenance/repair work by minimizing the time and cost of that work. This further works by minimizing human interferences and errors by synchronizing the system automatically and remotely. It is an automatic and time-saving tool. The entire record and 
monitored/controlled data of the building will improve the "resale" value of that building, and it is an essential tool to review errors by authorized jurisdiction. IoT usage encouragement might be as tax reduction, projects preferability, and future benefits. Law enforcement or binding legislation/regulations must set in advance to control, monitor, and punish intruders and hackers. The development process going in KSA, in both governmental and private sectors, is exceptionally qualified to practice CMMS configuration protocol where the megaprojects are the potential environment. The complexity of smart "Things" connectivity and intelligence need new engineering understanding concepts for the changes scientifically and socially.

\section{Data Availability Statement}

All data, models, and code generated or used during the study appear in the submitted article.

\section{References:}

1. Ashton, K. (2009). That 'Internet of Things' Thing. RFID Journal, 22, 97-114. Retrieved from https://www.rfidjournal.com/articles/view?4986

2. Bashir, M., \& Gill, A. (2016). Towards an IoT Big Data Analytics Framework: Smart Building Systems. In 2016 IEEE 18th International Conference on High Performance Computing and Communications; IEEE 14th International Conference on Smart City; IEEE 2nd International Conference on Data Science and Systems (HPCC/Smart City/DSS), Sydney, NSW, 2016, pp. 1325-1332.

DOI: 10.1109/HPCC-SmartCity-DSS.2016.0188

3. Becerik-Gerber, B., Jazizadeh, F., Li, N., \& Calis, G. (2012). Application Areas and Data Requirements for BIM-Enabled Facilities Management. Journal of Construction Engineering and Management (ASCE), 138, 431-442. https://doi.org/10.1061/(ASCE)CO.19437862.0000433

4. Bertino, E. (2016). Data Security and Privacy in the IoT. In Proceedings of the 19th International Conference on Extending Database Technology (2016), 1. https://doi.org/10.5441/002/edbt.2016.02

5. Brous, P., Marijn J., \& Herder, P. (2018). Internet of Things adoption for reconfiguring decision-making processes in asset management. Business Process Management Journal, 25, (3), 495511. Retrieved from: www.emeraldinsight.com/1463-7154.htm

6. Cadle, J., \& Donald, Y. (2008). Project management for information systems. 5th ed. Harlow: Pearson Prentice Hall. Retrieved 
from https://pdfs.semanticscholar.org/5613/2e7a7bb6ccc6b9a26d8fde 89d68fa0c18c9d.pdf

7. Chan, Y. (2015). Internet of Things Business Models. Journal of Service Science and Management, 8, 552-568. Retrieved from http://dx.doi.org/10.4236/jssm.2015.84056

8. Dandekar, A., Kanse, R., Magar, S., Tavhare, S., \& Katkar, A. (2017). IoT Based Green Building. International Journal of Computer Science and Mobile Computing, 6, (4), 439-443. Retrieved from: https://www.ijcsmc.com/docs/papers/April2017/V6I4201797.p df

9. Diaz, E. (2016). The new European Union General Regulation on Data Protection and the legal consequences for institutions. Journal Church, Communication and Culture, 1,(1), 206-239. Retrieved from https://doi.org/10.1080/23753234.2016.1240912

10. Eggleton, B. (2020). Smart Buildings: the critical questions answered. Worktechacademy. Accessed 20Dec2019:

https://www.worktechacademy.com/smart-buildings-questionsanswered/

11. Gartner, Inc. (2015). Gartner Says Smart Cities Will Use 1.6 Billion Connected Things. In 2016. December 7, 2015. Retrieved from www.gartner.com/newsroom/id/3175418. View in article

12. Gartner, Inc. (2019). Analysts to Explore How IoT Will Accelerate Digital Transformation Initiatives. The Gartner IT Symposium/Xpo, November 3-7, 2019 in Barcelona, Spain. Retrieved from https:/www.gartner.com/en/newsroom/press-releases/2019-08-29gartner-says-5-8-billion-enterprise-and-automotive-io

13. General Authority for Statistics (2017). Report of General Authority for statistics. Available online:

https://www.stats.gov.sa/sites/default/files/survey_of_construction_a ctivity_2017ar.pdf

14. Hamilton, L., Bezerra, N., Bezerra1, M., \& Filho, J. (2017). The internet of things sensors technologies and their applications for complex engineering projects: a digital construction site framework. Brazilian Journal of Operations \& Production Management, 14, 567-576. http://orcid.org/0000-0002-5368-3525

15. Jaafreh, A. (2018). The Effect Factors in the Adoption of Internet of Things (IoT) Technology in the SME in KSA: An Empirical Study. International Review of Management and Business Research (IRMBR).7, (1), 135-148. http://www.irmbrjournal.com/papers/1517914157.pdf

16. Jia, M., Srinivasan, R. \& Raheem, A. (2017). From occupancy to occupant behavior: An analytical survey of data acquisition 
technologies, modeling methodologies and simulation coupling mechanisms for building energy efficiency. Renewable and Sustainable Energy Reviews, 68, (1), 525- 540.

17. Kensek, K. (2014). Integration of Environmental Sensors with BIM: case studies using Arduino, Dynamo, and the Revit API. Informes de la Construcción, 66(536): e044, Doi: http://dx.doi.org/10.3989/ic.13.151.

18. Khakurel, J., Melkas, H. \& Porras, J. (2018). Tapping into the wearable device revolution in the work environment: a systematic review. Information Technology \& People, 31(3), 791-818 . https://doi.org/10.1108/ITP-03-2017-0076

19. KPMG (2016). Smart construction, how offsite manufacturing can transform our industry. April 2016. Kpmg.com/uk/ibc.Online: https://assets.kpmg/content/dam/kpmg/pdf/2016/04/SmartConstructio nReport.pdf

20. Lee, Y.T (1999). Information modeling from design to implementation. National Institute of Standards and Technology. https://tsapps.nist.gov/publication/get_pdf.cfm?pub_id=821265

21. Mztaloto, B., Ferreira, J. \& Cruz, N. (2019). LoBEMS-IoT for Building and Energy Management Systems. Electronics, 8(7), 763; https://doi.org/10.3390/electronics8070763

22. McGuckin, S. (2018). International construction market survey 2018. Accessed 12Septemper 2019.

https://www.turnerandtownsend.com/media/3352/internationalconstruction-market-survey-2018.pdf

23. McKinsey Global Institute (MGI) (2018). Smart Cities: Digital Solutions for a More LivableFuture.

https://www.mckinsey.com/ /media/mckinsey/industries/capital\%20 projects\%20and\%20infrastructure/our\%20insights/smart\%20cities\%2 0digital\%20solutions\%20for\%20a\%20more\%20livable\%20future/mg i-smart-cities-full-report.ashx

24. Mohammed, Z.K. \& Elmustafa, A. (2017). Internet of Things Applications, Challenges and Related Future Technologies. World Scientific News, 67(2), 126-148.

http://www.worldscientificnews.com/wpcontent/uploads/2017/01/W SN-672-2017-126-148-2.pdf

25. Neom. https://www.neom.com

26. NIST (2019). Considerations for Managing Internet of Things (IoT) Cybersecurity and Privacy Risks. National Institute of Standards and Technology Interagency or Internal Report 822844 pages (June 2019). Available from: https://doi.org/10.6028/NIST.IR.8228 
27. Oduyemi, O. \& Okoroh, M. (2016). Building performance modeling for sustainable building design. International Journal of Sustainable Built Environment, 5,( 2), 461-469.

https://doi.org/10.1016/j.ijsbe.2016.05.004

28. Oriwoh, E., Sant, P., \& Epiphaniou, G. (2013). Guidelines for Internet of Things deployment approaches - The Thing Commandments. Procedia Computer Science, 21, 122 - 131. https://doi.org/10.1016/j.procs.2013.09.018

29. OSRAM (2017). How to Calculate the Value Proposition of Smart Buildings. OSRAM 2017, Accessed 1Jan2020:

https://info.osram.us/blog/how-to-calculate-the-value-proposition-ofsmart-buildings

30. Rao, B.S., Chakravarthi, C. \& Jawahar, A. (20017). Industrial Control Systems Security and Supervisory Control and Data Acquisition (SCADA). International Journal for Modern Trends in Science and Technology, 3, 10: 109-118. Available online at http://www.ijmtst.com/vol3issue10.html

31. Reddy, K. P. (2011). BIM for building owners and developers: Making a business case for using BIM on projects. Indianapolis: Wiley.

32. Singh, J., Millard, C., Reed, C., Cobbe, J., \& Crowcroft, J. (2018). Accountability in the IoT: Systems, Law, and Ways Forward. Computer, 51(7), 54 - 65.DOI: 10.1109/MC.2018.3011052

33. The big 5 (2019). Sustainability and green building solutions at The Big 5. Accessed 20 September 2019: https://www.thebig5.ae/

34. Tzafestas, S. (2018). Ethics and Law in the Internet of Things World. Smart Cities, 1, 98-120;

https://doi.org/10.3390/smartcities1010006

35. Urie, M. (2019). The Internet of Things in Construction: Gardiner and Theobald. Accessed 1 June 2019:

https://marketintel.gardiner.com/bulletins/the-internet-of-things-inconstruction

36. Vermesan, O., \& Friess, P. (2014). Internet of Things Applications From Research and Innovation to Market Deployment. River Publishers Series in Communications. Available online:

http://www.internet-of-things-

research.eu/pdf/IERC_Cluster_Book_2014_Ch.3_SRIA_WEB.pdf

37. Wang, J., Xiangyu W., Shoua, W., Chong, H., \& Guo, J. (2016). Building information modeling-based integration of MEP layout designs and constructability. Automation in Construction 61, 134146. Accessed 15: August 2019:

http://dx.doi.org/10.1016/j.autcon.2015.10.003 
38. Weber, R. (2011). Accountability in the Internet of Things. Computer Law \& Security Review, 27, (2),133-138.

https://www.dhi.ac.uk/san/waysofbeing/data/governance-croneweber-2011a.pdf . doi:10.1016/j.clsr.2011.01.005

39. Woodhead, R., Stephenson, P. \& Morrey, D. (2018). Digital construction: From point solutions to IoT ecosystem. Automation in Construction. 93, 35-46. https://doi.org/10.1016/j.autcon.2018.05.004 40. Zhou, H., Wang, H., \& Zeng, W. (2018). Smart construction site in mega construction projects: A case study on island tunneling project of Hong Kong-Zhuhai-Macao Bridge. Frontiers of Engineering Management, 5(1): 78-87. https://doi.org/10.15302/JFEM 2018075 